\title{
Study on optimization of rake head density of suction hopper dredger based on bat algorithm and extreme learning machine
}

\author{
Guangjie $\mathrm{Hao}^{1}$, Menghong $\mathrm{Yu}^{1, *}$, and Zhen $\mathrm{Su}^{2}$ \\ ${ }^{1}$ School of Electronic and Information, Jiangsu University of Science and Technology, Zhenjiang \\ 212003, China \\ ${ }^{2}$ Department of Automation, College of Mechatronic Engineering and Automation, Shanghai \\ University, Shanghai 200072, China
}

Keywords: Rake head model, Density prediction, Bat algorithms, Output simulator, Extreme learning machine.

\begin{abstract}
The dredging output of suction dredger mainly comes from the suction density of the rake head. Accurate prediction of suction density is of great significance to improve the dredging output of suction dredger. In order to overcome the shortcomings of low accuracy and poor real-time performance of the current inhalation density prediction methods, a bat algorithm is proposed to optimize the inhalation density prediction method of extreme learning machine. The bat algorithms for optimizing extreme learning machines prediction model is constructed based on the measured construction data of "Xinhaifeng" Yangtze Estuary, and compared with other prediction models. Finally, the bat algorithms for optimizing extreme learning machines model is used to build the output simulator of inhalation density. Compared with the actual construction, the selection of control parameters is analyzed when the output of inhalation density is the best. Experients show that bat algorithms for optimizing extreme learning machines prediction has high accuracy and good stability, and can provide scientific and effective reference for yield prediction and construction guidance.
\end{abstract}

\section{Introduction}

In the dredging process of the dredger, the dredging output is mainly caused by the excavation of the rake head. Therefore, the dredging performance of the rake head directly affects the production efficiency of the suction dredger. The production of rake head is mainly determined by the suction density of rake head. Therefore, it is especially important to establish a suitable rake head model to study the inhalation density. ${ }^{[1]}$ The known physical model of the rake head is set under ideal conditions, such as keeping the soil type unchanged, and the type of rake head used is unchanged. ${ }^{[2,3]}$ However, in the actual

\footnotetext{
*Corresponding author: 2079433439@qq.com
} 
dredging construction; the above conditions cannot be satisfied; in addition, the mud mixture formation process is complicated in the rake head, and the establishment of the physical model of the rake head also needs to consider many unknown parameters from the soil, such as permeability and on-site density. ${ }^{[2,4]}$ In summary, it is difficult to establish an effective rake head model through physical analysis.

In response to the study of the rake head model, some scholars have proposed the use of data for black box modeling. In the literature [4], the least square method is used to fit the inhalation density. This method has certain limitations. When there are new working conditions, the collected data needs to be retrained. In the literature $[5,6]$, the genetic BP is used to predict the inhalation density. This method has the disadvantages of complicated parameter setting, long training time and low prediction accuracy.

At present, artificial intelligence, big data and other technologies are widely used in various industries, and they have achieved very good results. In view of the research on the production of rake head, this paper uses the extreme learning machine (ELM) algorithm to analyze the data black box of rake head. The Extreme Learning Machine (ELM) algorithm only needs to set the number of hidden layer neurons. It can obtain the unique optimal solution without adjusting the neuron weights and thresholds. Compared with other traditional neural networks, it has strong learning ability., the calculation speed is fast, easy to converge and so on; ${ }^{[7,14]}$ in the commodity futures price ${ }^{[8]}$, power load ${ }^{[9]}$ forecast have achieved good results. In order to improve the generalization performance of the Extreme Learning Machine (ELM) model, some literatures use particle swarm optimization algorithm ${ }^{[10]}$ and genetic algorithm ${ }^{[11]}$ to optimize the connection weight and hidden layer threshold of ELM. However, the particle swarm optimization algorithm has the disadvantages of limited search ability and low learning precision, which leads to the improvement of the complexity of the algorithm. The use of genetic algorithms also has limited space search ability, and it is easy to produce premature and local optimal problems. In this paper, the bat algorithm is used to optimize the extreme learning machine (ELM). This method is easy to realize the dynamic conversion between the global search and the local search. Compared with other optimization algorithms, the structure is simple, the parameter setting is less, and the method is better Convergence performance.

\section{Rake head production analysis}

\subsection{Dredging data analysis}

During the dredging construction of the suction dredger, the factors affecting the dredging output can be divided into the excavation volume of the rake head and the overflow of the mud hopper. The more the digging volume is, the more the hopper are loaded; the less the cabin overflow, the higher the dredging output. Since the suction flow can be controlled by the mud pump, the most representative factor for the amount of digging is the suction density of the rake head. There are many factors affecting the suction density of the rake head. Generally, there are high pressure flushing, excavation depth, mud pump speed, wave compensator stroke, lip pressure, etc. These influence factors interact with each other. According to the actual operation experience of dredging construction personnel and the literature, ${ }^{[1,5,12]}$ this paper chooses to build the suction density with mud pump speed, ship speed, high pressure flushing and steaming head angle and suction flow 5 inputs model prediction. 


\subsection{Data preprocessing}

The dredging data set contains the whole process from navigation, dredging and dumping. The data set required for the experiment is located in the dredging stage. The data set needs to be effectively organized. For example, the boundary conditions are set according to the construction parameters (considering the inhalation of the rake head) The density is greater than $1 \mathrm{ton} / \mathrm{m}^{3}$, the ship speed and the mud pump speed during normal construction), and the invalid data is eliminated.

Dredging data is collected from various sensors and controllers for suction dredgers. These data vary greatly from one another and vary in dimension. In order to avoid the influence of the data range size on the prediction results, the original data is dimensionless, normalized and standardized.

\section{Introduction to related algorithms}

\subsection{Extreme learning machine}

Assume that the vector of the collected data training samples is $\left\{\left(x_{1}, y_{1}\right),\left(x_{2}\right.\right.$, $\left.\left.y_{2}\right),\left(x_{3}, y_{3}\right), \cdots \cdots\left(x_{i}, y_{i}\right)\right\}$, where $x_{i} \in \mathrm{R}^{\mathrm{d}}, y_{i} \in \mathrm{R}^{\mathrm{m}}, \mathrm{i} \in\{1,2,3, \cdots \cdots \mathrm{N}\}$.

Assuming that the number of ELM hidden layer nodes is $\mathrm{L}$ and the hidden layer node output function is $\mathrm{k}(\mathrm{x})$, then its model is:

$$
\left\{\begin{array}{l}
\sum_{i=1}^{L} \beta_{i} k\left(a_{i} x_{1}+b_{i}\right)=t_{1} \\
\sum_{i=1}^{L} \beta_{i} k\left(a_{i} x_{2}+b_{i}\right)=t_{2} \\
\sum_{i=1}^{L} \beta_{i} k\left(a_{i} x_{3}+b_{i}\right)=t_{3} \\
\cdots \\
\sum_{i=1}^{L} \beta_{i} k\left(a_{i} x_{d}+b_{i}\right)=t_{m}
\end{array}\right.
$$

where $a_{i}=\left[a_{i 1}, a_{i 2}, a_{i 3}, \ldots, a_{i d}\right]^{T}$ is the weight between the $\mathrm{i}$-th single hidden layer node and the input node, $b_{i}$ is the threshold of the i-th hidden layer node in the network; $\beta_{i}=\left[\beta_{i 1}, \beta_{i 2}, \beta_{i 3}, . . \beta_{i L}\right]^{T}$ is the right of the $\mathrm{i}$-th hidden layer node and the output node The value, $\mathrm{k}(\mathrm{x})$ is the neuron matrix, and the expression (1) is converted into the expression of the matrix:

$$
T=K \beta
$$

where $\beta$ can be solved by least squares

$$
\beta=K^{+} T
$$




\subsection{Bat algorithm optimization ELM model construction}

The Bat Algorithm (BA) is an efficient bio-heuristic algorithm developed by Xin-SheYang in 2010. Its principle is to simulate bats using sonar to detect prey. ${ }^{[9,14]}$ The implementation process is as follows.

1. Set the initial number of bats to $\mathrm{n}$, the maximum pulse volume to $\mathrm{A}_{\max }$, the maximum pulse rate to $\mathrm{R}_{\max }$, the search pulse frequency range $\left[F_{\min }, F_{\max }\right]$, the volume attenuation rate to $\beta$, the search frequency enhancement factor to $\mu$, and the maximum iteration number to $\mathrm{N}_{\max }$.

2. Randomly initialize the position $X_{i}$ of the bat, and find the optimal solution $X_{b e s t}$ by judging the current fitness.

3. The search pulse frequency, speed and position of each generation are updated in the following ways:

$$
\begin{aligned}
& F_{i}=F_{\text {min }}+k\left(F_{\text {max }}-F_{\text {min }}\right) \\
& v_{i}^{t}=v_{i}^{t-1}+F_{i}\left(X_{i}^{t}-X_{\text {best }}\right) \\
& X_{i}^{t}=X_{i}^{t-1}+v_{i}^{t}
\end{aligned}
$$

where $\mathrm{k}$ is a random number between 0 and $1, F_{i}$ is the search pulse frequency of the $\mathrm{i}$-th bat, $F_{i} \in\left[F_{\min }, F_{\max }\right] ; X_{i}^{t}$ and $X_{i}^{t-1}$ represent the position of the $\mathrm{i}$-th bat at time $\mathrm{t}$ and $\mathrm{t}-1$, respectively. And the speed at $v_{i}^{t}$ and $v_{i}^{t-1}$ at which it corresponds.

4. A random number rand is generated. If rand is greate $r_{i}$, the current solution is randomly perturbed, a new solution is generated, and the new solution is processed out of bounds.

5. Generate a random number rand, if rand is less than $A_{i}$ and $F\left(X_{i}\right)$ less than $F\left(X_{\text {best }}\right)$, accept the new solution above, and update $r_{i}$ and $A_{i}$ as follows:

$$
\begin{array}{r}
A_{i}^{t+1}=\beta A_{i}^{t} \\
r_{i}^{t+1}=R_{\max }[1-\exp (-\mu t)]
\end{array}
$$

where: $\beta$ and $\mu$ have a value range of $(0,1)$, and are generally set to 0.9 .

The fitness values of $\mathrm{n}$ bats are sorted to find the current optimal solution.

7. Repeat steps 2-6 until the maximum number of iterations is met, and the optimal connection weight and hidden layer threshold are obtained.

Finally, the optimal connection weight and the hidden layer threshold are output to the ELM, the test set is then used to predict the outputs of the rake head. The construction of the BA-ELM prediction model is shown in Fig.2. 


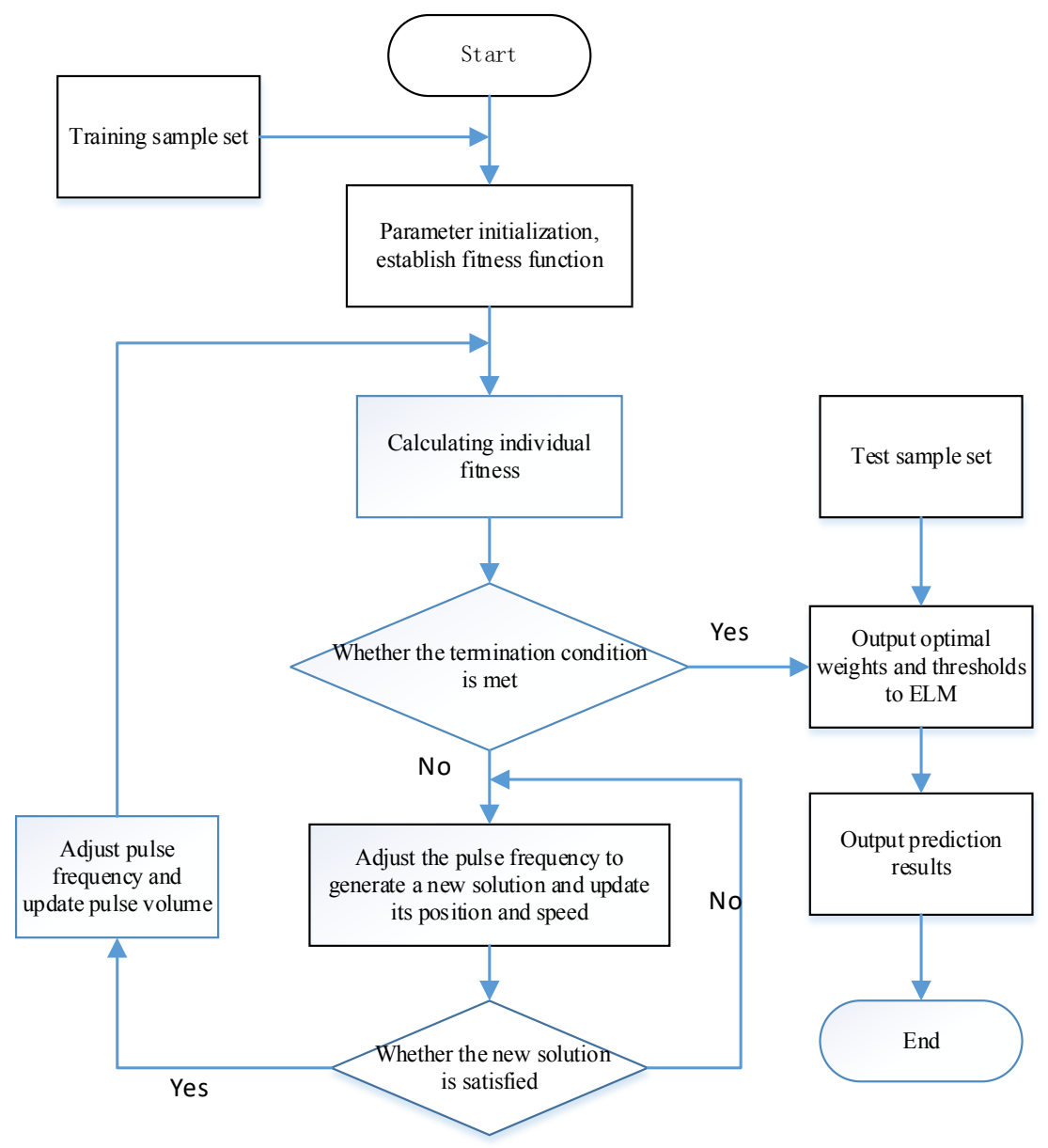

Fig. 2. BA-ELM optimization model flow chart.

\section{Simulation experiments and analysis}

\subsection{Data source}

The experimental data comes from the dredging project of the Yangtze River estuary of the 'Xinhaifeng' suction dredger. Data points are collected every 30 seconds. After the data is preprocessed, 4300 data of 12 cycles are organized, 10 cycles are taken as training data, and the remaining 2 cycles are test data. Fig. 3 shows the distribution of the original suction density data of the suction dredger in 150 minutes at 300 sampling points in one cycle. The data points during the construction phase are distributed at [50,240] for a total of 190 sampling points for 95 minutes. 


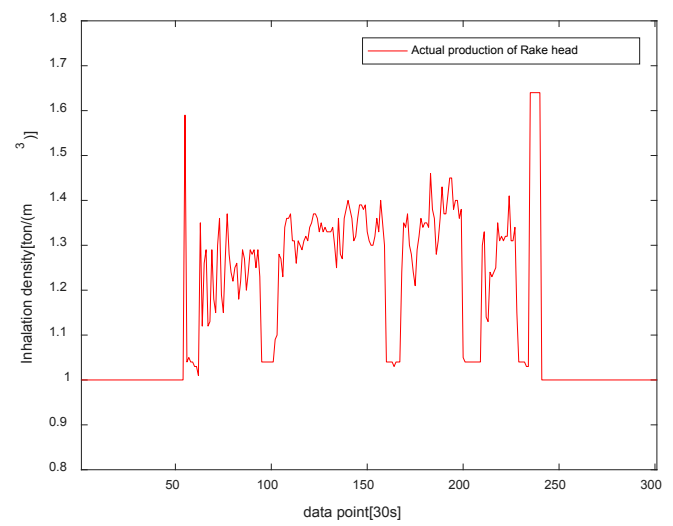

Fig. 3. Raw data distribution map.

In order to reflect the prediction performance of BA-ELM model, BP, ELM, particle swarm optimization (PSO-ELM) and genetic algorithm optimization (GA-ELM) were added to compare the experimental simulations, and the average error was used as the evaluation index.

\subsection{Analysis of experimental results}

In this experiment, the number of BP input layer neurons is 5 , the number of hidden layer neurons is 12, and the output layer is 1 . The number of ELM hidden layer neurons is set to 1200. The bat algorithm initializes the population to 30 , the pulse loudness $A_{\max }$ is 0.6 , the maximum pulse rate is $R_{\max } 0.6$, the search pulse frequency range [0,2], and the maximum number of iterations is set to 1000 . The simulation results are shown in Figure 4.1 and Figure 4.2.
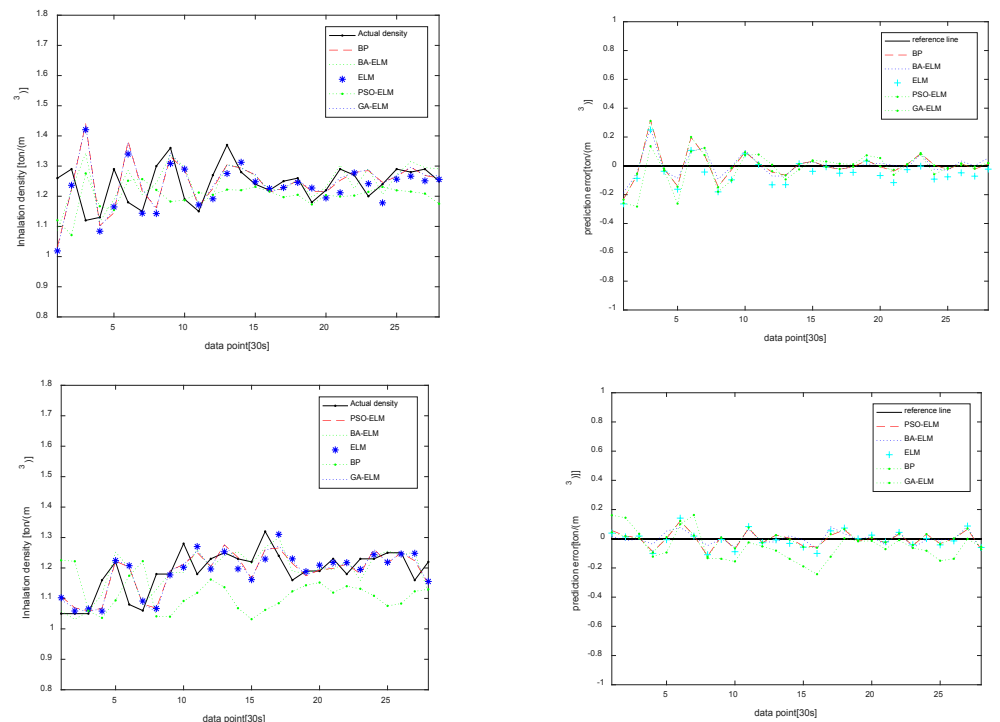

Fig. 4.1. Cycle 1 inhalation density prediction.

Top left, Left head inhalation density model; top .right, Left head prediction error; bottom left, Right head inhalation density model; bottom right, Right head prediction error 

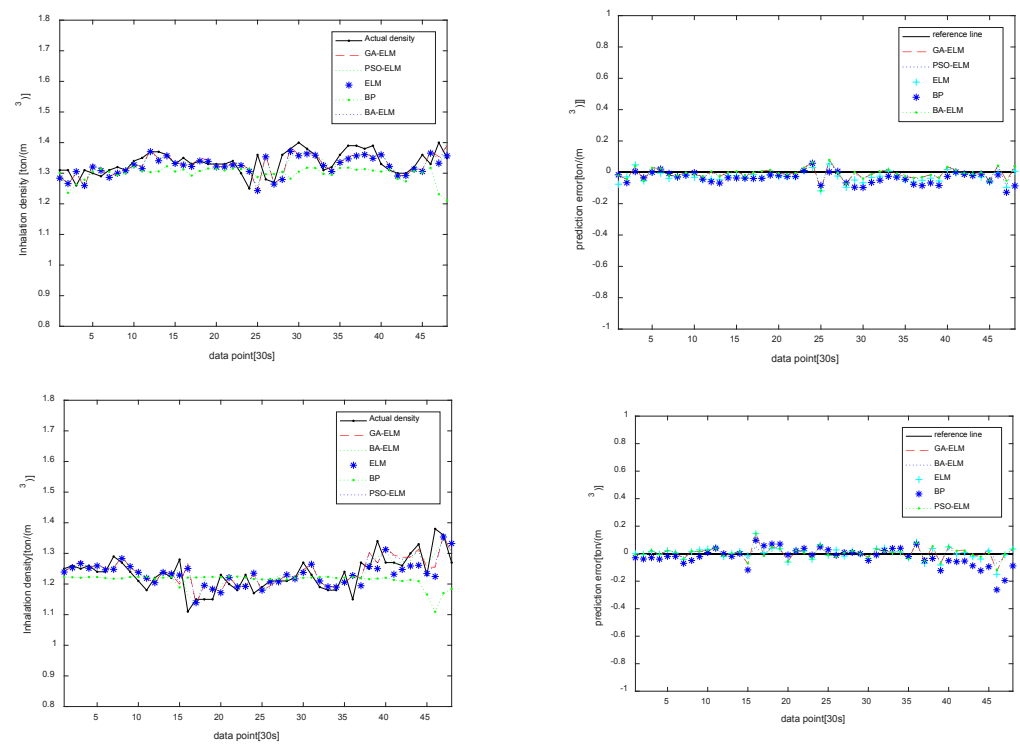

Fig. 4.2. Cycle 2 inhalation density prediction.

Top left, Left head inhalation density model; top .right, Left head prediction error; bottom left, Right head inhalation density model; bottom right,Right head prediction error

Figure 4.1 shows the prediction results and error comparison diagrams of the five prediction methods for the first cycle of the suction dredger. The first cycle is 28 sampling points, which is the 14-minute output of rake head. It can be found from Fig. 4.1 that BP has the worst prediction effect, ELM learning speed is faster, and generalization performance is better than BP; ELM prediction effect is improved by ELM optimization. Among the prediction effects of GA-ELM, PSO-ELM and BA-ELM, the BA-ELM model has the smallest error and the best prediction effect. Fig.4.2 is a second cycle prediction rendering (a total of 48 sampling points for a total of 24 minutes). The experimental results are consistent with Fig.4.1 .The average error of the five prediction methods is calculated, and the average error of each period prediction model is shown in Tab.1.

Table 1. Average error of each period prediction model.

\begin{tabular}{|c|c|c|c|c|c|}
\hline Predictive model & BP & ELM & GA-ELM & BA-ELM & PSO-ELM \\
\hline Cycle 1 left & 0.0641 & 0.0635 & 0.0629 & 0.0571 & 0.0617 \\
\hline Cycle 1 right & 0.0769 & 0.0462 & 0.0428 & 0.0351 & 0.0412 \\
\hline Cycle 2 left & 0.0841 & 0.0308 & 0.0261 & 0.0257 & 0.0283 \\
\hline Cycle 2 right & 0.0591 & 0.0331 & 0.0313 & 0.0288 & 0.0301 \\
\hline
\end{tabular}

As can be seen from Tab.1, the average error of ELM prediction is less than BP, so the prediction effect of ELM is better than BP. After optimizing for ELM, its prediction performance is improved, and the average error of BA-ELM is the smallest. Therefore, the predicted performance is the best.

The trained BA-ELM model was used to simulate the production of rake head, and the construction parameters were optimized. Set the mud pump speed, ground angle and high pressure flush to a fixed value according to the required suction density of the hopper, 
control the inhalation density to $\left[1 \mathrm{ton} / \mathrm{m}^{3}, 1.4 \mathrm{ton} / \mathrm{m}^{3}\right]$, and set the suction flow rate to [2 $\mathrm{m}^{3} / \mathrm{s}, 10 \mathrm{~m}^{3} / \mathrm{s}$ ] and the ship speed set to $[0 \mathrm{kn} / \mathrm{h}, 6 \mathrm{kn} / \mathrm{h}$ ] for visual analysis, the following figure shows the simulation of the rake head output in two cycles, as shown in Fig. 5.
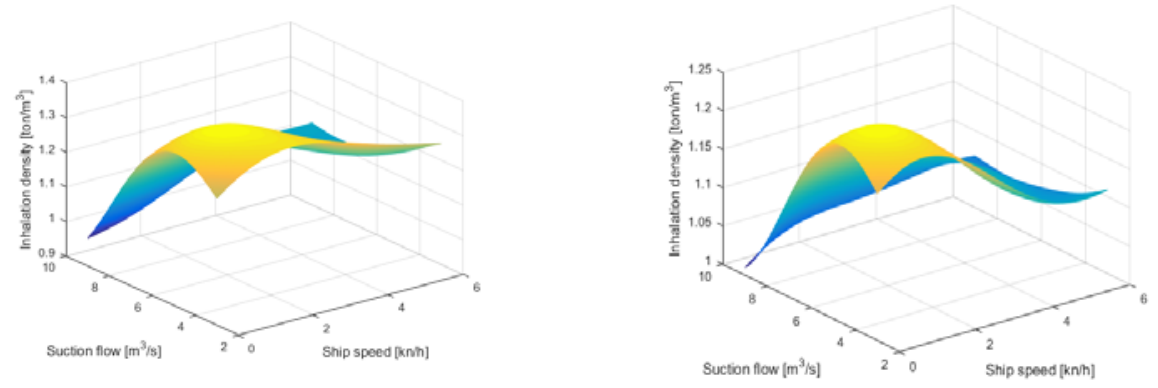

Fig. 5. Inhalation Density production Simulation:left:cycle1, right:cycle 2.

In the cycle 1 of Fig. 5, the mud pump speed was set at $200 \mathrm{rpm}$, the ground angle was set to 7 degrees, and the high pressure flush was 0.52 Bar. The seawater density is 1000 $\mathrm{kg} / \mathrm{m} 3$; in cycle 2 , the mud pump speed is set at $200 \mathrm{rpm}$, the ground angle is set to 2 degrees, and the high pressure water is 0.52 Bar. It can be seen from Fig. 5, the better the inhalation density (the higher the color brightness), the higher the rake head output, the suction flow rate is generally in the range of $7 \mathrm{~m} / \mathrm{s}$ to $8.5 \mathrm{~m} / \mathrm{s}$, and the ship speed between 1 to 2.6 knots. In addition, it can be seen from Fig. 5 that the selection of the ground angle has a very significant influence on the output of the rake head. Comparing the above-mentioned rake head production simulation map with the actual dredging construction process parameters, the suction flow rate and the ship speed value are consistent with the actual construction conditions.

\section{Conclusion}

Based on the analysis of inhalation density in the model of raking head output, In view of previous research, a method of bat algorithm optimization extreme learning machine is proposed to predict the inhalation density. The experimental simulation analysis proves that the prediction effect of this method is obviously better than other methods, and it can be used as a technical means for forecasting the production of rake head.

By using the BA-ELM model to establish the rake head production simulator, the optimization of the construction process parameters can be realized. This method can be used to guide the dredging construction on site and improve the dredging production efficiency.

This research is financially supported by China Communications Construction Co., Ltd. project number: (2035151801)

\section{References}

1. Zhen S, Cao D, Jian S . Modeling and Simulation of Drag head on Trailing Suction Hopper Dredger[C]// International Conference on Frontier Computing. Springer, Singapore, 2018.

2. $\mathrm{Z} \mathrm{Su}, \mathrm{ZX} \mathrm{Zhou}, \mathrm{MH} \mathrm{Yu}$, et al.Online Estimation of Soil Grain Diameter during Dredging of Hopper Dredger Using Continuous-Discrete Feedback Particle Filter[J].Sensors and Materials, Vol.31,No.3 (2019) 953-968:3-7 
3. BRAAKSMA J. Model-based control of hopper dredgers [D] . Delft: Delft University of Technology, 2008:1-21

4. Wangli. Intelligent data analysis and control of a hopper dredger[D]. Holand :Delft University of Technology. 2006:42, 79, 169.

5. Cao Dian, Su Zhen, ye Shuxia. Prediction of rake head density of dredger based on Genetic BP neural network [J]. Water transport in China (the second half of the month),2016(10).

6. Su Z, Fu J, Sun J . A Genetic Neural Network Approach for Production Prediction of Trailing Suction Dredge[J]. 2017.

7. Huang G B , Zhou H , Ding X, et al. Extreme Learning Machine for Regression and Multiclass Classification[J]. IEEE Transactions on Systems, Man and Cybernetics, Part B (Cybernetics), 2012, 42(2):513-529.

8. Feng Jiang, Jiaqi He, Zhigang Zeng. Pigeon-inspired optimization and extreme learning machine via wavelet packet analysis for predicting bulk commodity futures prices[J]. Science China Information Sciences, 2019, 62(7):70204.

9. Kong Lingchun, sun qiongqiongqiong, Yang Zhaofeng. Power load forecasting model of bat algorithm optimized learning machine [J]. Journal of Liaoning University of engineering and Technology (NATURAL SCIENCE EDITION),2016,35(01):89-92.

10. $\mathrm{Xu}$ Aidong, Li haofei, Cheng Lefeng, et al. Pca-pso-elm power supply reliability prediction model [J]. Journal of Harbin Engineering University2018,39(06):1116-1122.

11. Lu Fangcheng, Liu Yi, Xie Qing, et al. Short term power load forecasting based on improved genetic algorithm to optimize limit learning machine $[\mathrm{J}]$. Journal of North China Electric Power University (NATURAL SCIENCE EDITION),2018,45(06):1-7.

12. Zhen Su, Wei Yuan. Parameter Estimation of Trailing Suction Hopper Dredger Dredging Model by GA[M]// Frontier Computing. Springer Singapore, 2016.:1-7.

13. Huynh H T, Won Y, Kim J J. AN IMPROVEMENT OF EXTREME LEARNING MACHINE FOR COMPACT SINGLE-HIDDEN-LAYER FEEDFORWARD NEURAL NETWORKS[J]. International Journal of Neural Systems, 2008, 18(05):433-441.

14. Xin - She Yang, Gandomi A H . Bat algorithm: a novel approach for global engineering optimization[J]. Engineering Computations, 2012, 29(5):464-483. 